\title{
Determinación por espirometría de volúmenes y capacidades pulmonares en sujetos exfumadores
}

\author{
Pulmonary volumes and capacities \\ determined by spirometry in ex-smokers
}

Javier Eliécer Pereira-Rodríguez ${ }^{1}$, Devi Geesel Peñaranda-Flórez², Pedro Pereira-Rodríguez ${ }^{3}$, Ricardo Pereira-Rodríguez ${ }^{4}$, Juan Camilo Quintero-Gómez ${ }^{5}$, Roberto Aldair Mauricio-Reynoso ${ }^{6}$

\section{Resumen}

INTRODUCCIÓN: las enfermedades respiratorias son uno de los principales problemas de salud pública para la economía mundial. Uno de los principales factores de riesgo es el tabaquismo. Las pruebas de función pulmonar, como la espirometría, nos permiten determinar las alteraciones del sistema respiratorio y su evolución a partir de valores cuantitativos.

OBJETIVO: determinar mediante espirometría los volúmenes y las capacidades pulmonares de sujetos exfumadores.

MATERIALES Y MÉTODOS: estudio transversal, descriptivo y observacional con 848 sujetos de ambos géneros, mayores de 16 años, exfumadores de más de un año de abandono, con una edad promedio de 36,62 $\pm 10,15$ (hombres: 36,62 $\pm 10,13$ frente a mujeres: 40,36 \pm 15,86). La función pulmonar se determina por espirometría con el equipo Nuevo Spirobank $\mathrm{II}{ }^{\circledR}$, que permite determinar, entre otros, la capacidad vital forzada (FVC), la capacidad vital (VC) y el volumen máximo espirado en el primer segundo de una espiración forzada $\left(\mathrm{FEV}_{1}\right)$.

RESULTADOS: 848 sujetos, el 3,3\% mujeres y el 96,7\% hombres, con una edad de $36,62 \pm 10,15$ años, talla de $169,71 \pm 6,77 \mathrm{~cm}$ y un peso de $72,66 \pm 12,56$. La abstinencia del tabaco fue de 4,34 $\pm 5,27$ años, en un consumo diario relativo de 5,76 \pm 7,28 cigarrillos por día. La relación $\mathrm{FEV}_{1} / \mathrm{FVC}$ fue de $87,28 \%$, el $\mathrm{FEV}_{1}$ de 3,81 L $\pm 0,02$ y la FVC de 4,38 L \pm 0,02. Del total de los sujetos, 754 (88,92\%) tenían valores de FEV/FVC normales y $54(6,37 \%)$ presentaban obstrucción pulmonar leve tras haber dejado el hábito de consumo de tabaco.

CONCLUSIONES: el consumo del tabaco reduce los parámetros de las capacidades y los volúmenes pulmonares en personas, independientemente de las características antropométricas. El abandono del hábito tabáquico puede prevenir las alteraciones en la función pulmonar.

Palabras clave: espirometría, función pulmonar, exfumadores.

\section{Abstract}

INTRODUCTION: respiratory diseases are one of the main public health problems for the global economy. One of the main risk factors is smoking. Pulmonary function tests, such as spirometry, allow us to determine the alterations of the respiratory system and its evolution from quantitative values.

OBJECTIVE: the purpose of this research is to determine lung volumes and capacities in ex-smokers through spirometry.
${ }^{1}$ Fisioterapeuta, especialista en rehabilitación cardiopulmonar, maestrante en Ciencias de la Salud y en innovación educativa, docente universitario; Puebla, México.

${ }^{2}$ Fisioterapeuta, especialista en neurorrehabilitación, magíster en dificultades del aprendizaje. Consultorio Independiente Privado; Puebla, México.

${ }^{3}$ Médico general, residente en medicina de urgencias y cuidado del paciente en estado crítico. Fundación Universitaria de Ciencias de la Salud; Bogotá, Colombia.

${ }^{4}$ Médico general, Unidad de Cuidados Intensivos. Universidad Rafael Núñez; Cúcuta, Colombia.

${ }^{5}$ Estudiante de Fisioterapia, Universidad de Santander; Cúcuta, Colombia.

${ }^{6}$ Estudiante de Fisioterapia; Puebla, México.

Grupo de investigación Alétheia.

Autor de correspondencia

Javier Eliécer Pereira-Rodríguez.

Correo electrónico:

jepr87@hotmail.com

Recibido: 26/04/2020

Aceptado: 01/05/2020 
MATERIALS AND METHODS: Cross-sectional, descriptive and observational study with 848 subjects of both genders, older than 16 years of age, ex-smokers older than one year of abandonment, with an average age of $36.62 \pm 10.15$ (men: $36.62 \pm 10.13$ vs. women: $40.36 \pm 15.86)$. The pulmonary function is determined by spirometry with the New Spirobank II ${ }^{\circ}$ equipment, which allows determining, among others, the forced vital capacity $(\mathrm{FVC})$, vital capacity $(\mathrm{VC})$ and forced expiratory volume in one second $\left(\mathrm{FEV}_{1}\right)$.

RESULTS: 848 subjects, $3.3 \%$ women and $96.7 \%$ men, with an age of $36.62 \pm 10.15$ years, height of $169.71 \pm 6.77 \mathrm{~cm}$, and a weight of $72.66 \pm 12,56$. Tobacco abstinence of 4.34 years \pm 5.27 in a relative daily consumption of $5.76 \pm 7.28$ cigarettes per day. $\mathrm{FEV}_{1} / \mathrm{FVC}$ of $87.28 \%, \mathrm{FEV}_{1}$ of $3.81 \mathrm{~L} \pm 0.02$ and $\mathrm{FVC}$ of $4.38 \mathrm{~L} \pm 0.02$. Of the subjects, $754(88.92 \%)$ had normal $\mathrm{FEV}_{1} / \mathrm{FVC}$ values and $54(6.37 \%)$ had mild pulmonary obstruction after having quit smoking.

CONCLUSIONS: the consumption of tobacco reduces the parameters of lung capacities and volumes in people independent of the anthropometric characteristics. The abandonment of smoking can prevent alterations in lung function indifferent from the moment it is suspended.

Keywords: Spirometry; Pulmonary Function; Ex-smokers.

\section{Introducción}

El sistema respiratorio es el responsable de tomar el oxígeno de la atmósfera y transportarlo hasta el sistema circulatorio; de la misma manera, conduce el dióxido de carbono, producto desecho del metabolismo celular, desde el interior hasta el exterior, regulando las concentraciones de $\mathrm{pH}$ y oxigenando la sangre venosa mixta. Así, es importante reconocer la fisiología pulmonar para poder interpretar las pruebas de función pulmonar (PFP) (1).

Entendiendo la fisiología del sistema respiratorio, la ventilación es el movimiento del aire desde el ambiente externo hasta los alvéolos pulmonares, donde los pulmones generan presión negativa para poder distribuir el oxígeno hasta estas unidades funcionales más pequeñas (2). El intercambio de gases hace referencia al movimiento de oxígeno en el sistema circulatorio y el gas carbónico en los alvéolos que será eliminado.

Las enfermedades respiratorias son uno de los problemas de salud con mayor impacto sobre la economía mundial, pues constituyen 5 de las 30 causas más comunes de mortalidad y causan más del $10 \%$ de todos los años de vida perdidos ajustados por discapacidad (AVAD) (3). Para el año 2015, la Organización Mundial de la Salud (OMS) estimó que 65 millones de personas padecían de enfermedad pulmonar obstructiva crónica (EPOC) de moderada a grave, de los cuales 3 millones mueren cada año aproximadamente, lo que la convierte en la tercera causa de muerte en todo el mundo solo después de las enfermedades cardiovasculares (4).
Uno de los mayores responsables de las enfermedades respiratorias durante muchos años ha sido el tabaco; de los 4000 productos químicos que contiene, 250 son nocivos y más del $50 \%$ están asociados al cáncer de pulmón. Cada año mueren aproximadamente 7 millones de personas por el consumo de tabaco, que son cerca de la mitad de todos los consumidores; el $80 \%$ de los más de mil fumadores son de países de ingresos bajos y medios (5). Es así como las PFP son indispensables para poder evaluar el estado de la capacidad ventilatoria y hacer el seguimiento de las enfermedades respiratorias a partir del análisis de gases (2).

Unos de los principales motivos por los cuales los pacientes se someten a PFP es la disnea (6). Estos sistemas de valoración de la función pulmonar permiten determinar y cuantificar el estado de la capacidad respiratoria a partir de los valores de los volúmenes pulmonares. Además de determinar anomalías sobre la función pulmonar, las PFP también permiten realizar un seguimiento sobre la evolución de las enfermedades respiratorias, o aquellas enfermedades neuromusculares y cardiovasculares que pueden afectar los volúmenes a nivel pulmonar (7).

De esta manera, las pruebas que permiten evaluar la función pulmonar pueden incluir diferentes estudios. La espirometría, por ejemplo, es la más usada y fácil de reproducir en el ámbito clínico en los pacientes con una alteración del sistema respiratorio. Esta prueba permite medir los volúmenes pulmonares y la capacidad de difusión de gas carbónico, y establece la rela- 
ción flujo-volumen (8). Para su realización, una de las indicaciones más importantes consiste en que no haya existido un uso previo de broncodilatadores ni consumo de tabaco para el día de la prueba, lo que minimiza el riesgo de alterar los valores fisiológicos de la respuesta pulmonar (6).

Los valores tomados en la realización de la espirometría son difíciles de calcular con un alto grado de confiabilidad. Dado esto, se han establecido unos factores de referencia para evitar el sesgo o la confusión de los valores calculados en las PFP (9). Dentro de estos factores tenemos el peso, la estatura, el género y la edad; otros, como el ciclo menstrual, el diámetro torácico, el tamaño de la tráquea y el ritmo circadiano son sujetos de nuevas investigaciones en torno a la función pulmonar (10-21).

Algunos otros factores, como las características sociodemográficas, las conductas y los hábitos de vida (tabaquismo, nutrición, alcoholismo y actividad física), las enfermedades, la genética y el desarrollo vital son aspectos importantes en la realización de las PFP y su análisis, que ya resulta complejo (22-25). De esta manera, el objetivo de esta investigación fue determinar los volúmenes y las capacidades pulmonares en sujetos exfumadores mediante una espirometría.

\section{Material y métodos}

\section{Diseño}

Estudio de descriptivo observacional de corte transversal, con análisis cuantitativo por conveniencia. Se recopiló la información de 848 pacientes de Cúcuta, Colombia, sin un diagnóstico de enfermedad pulmonar, atendidos entre 2016-2018.

\section{Sujetos}

Se obtuvo una muestra representativa de 848 pacientes, quienes firmaron previamente un consentimiento informado para la realización de las pruebas, recolección y uso de la información para el estudio. Se incluyeron sujetos sin diagnóstico de enfermedad pulmonar; hombres o mujeres mayores de 16 años. Por otra parte, los sujetos debían ser exfumadores con más de un año de abstinencia, con historia de consumo diario de cigarrillos.
No hicieron parte del estudio aquellos pacientes menores de 16 años, fumadores, exfumadores con menos de un año de retiro tabáquico. Además de esto, se excluyeron pacientes con historia clínica de neumotórax, enfermedad cardiovascular inestable (angina inestable, infarto agudo de miocardio reciente), tromboembolismo pulmonar, hemoptisis, aneurismas con riesgo de ruptura, desprendimiento de retina o cirugía ocular reciente, cirugía reciente de tórax o abdomen.

También se excluyeron aquellos participantes con traqueotomía, problemas bucales o faciales, náuseas incontrolables al introducir la boquilla, incontinencia de esfuerzo, estado físico o mental deteriorado, falta de colaboración o quienes no comprendieran bien la maniobra.

\section{Materiales}

Previo a la realización de la espirometría, se recolectó la información de las historias clínicas mediante un cuestionario diseñado para la investigación. Este permitió identificar variables según la edad (mayores a 16 años) y antecedentes de consumo de tabaco (mayor a un año de retiro tabáquico). Sobre el hábito y consumo de tabaco, se identificó: años y cantidad de consumo diario.

Para la evaluación de la función pulmonar se utilizó el espirómetro Nuevo Spirobank II ${ }^{\circledR}$, que permite determinar los valores de FVC y VC, pre y pos, con los principales parámetros habilitados $\left(\mathrm{FEV}_{1}, \mathrm{FEV} 1 \%\right.$, flujo espiratorio máximo [PEF], flujos espiratorios instantáneos [FEF] 25-75 \%, tiempo de espiración forzada [FET], volumen extrapolado [EVoL], capacidad vital inspiratoria [IVC], capacidad inspiratoria [IC] y volumen de reserva espiratoria [ERC]). Además, este identifica la edad pulmonar (ELA) para medir inmediatamente los beneficios después de haber dejado de fumar, indicador semafórico para un diagnóstico inmediato, y realiza un análisis de las pruebas efectuadas. También cuenta con una medición del flujo de $\pm 16 \mathrm{~L} / \mathrm{s}$, exactitud del volumen de $\pm 3 \%$ o $50 \mathrm{~mL}$, exactitud del flujo de $\pm 5 \%$ o $200 \mathrm{~mL} / \mathrm{s}$ y una variación menor a $0,2 \mathrm{~L}$.

\section{Procedimientos}

A todos los sujetos se les realizó un cuestionario para consignar la información personal y validar los 
datos correspondientes; además, para describir la frecuencia y la cantidad de tabaco según días y unidades. Posteriormente, se les explicó en qué consiste la PFP y cuál es el fin de la valoración. La prueba se realiza por un profesional con la formación necesaria para llevar a cabo los distintos procedimientos de espirometría bajo fundamentos en fisiopatología y enfermedades respiratorias, cumpliendo los mínimos estándares de calidad.

De manera previa a la realización de la prueba, se tomaron los valores para la talla y el peso del paciente. El peso se obtuvo con el sujeto en ropa ligera y la talla se recolectó con el sujeto descalzo, con el cuerpo estirado y la cabeza en plano de Frankfort. Posterior a esto, los participantes debían reposar 15 minutos antes de iniciar la prueba, en la que se les brindó indicaciones sobre la posición de la boca, los dientes y la lengua para evitar que se obstruyera la entrada de aire; debían mantener una postura erguida sobre el espaldar de una silla, sobre la que su cuerpo reposara durante la prueba, sin cruzar las piernas y con los pies adosados al suelo. La espirometría se realizó en un equipo con neumotacógrafo calefaccionado (SensorMedics), calibrado con una jeringa de 3 L cada día.

Los pacientes debían respirar a través de la boca, con los ojos cerrados y los labios alrededor para evitar la salida del aire; se recurrió al uso de pinzas nasales para asegurar la salida de aire por la nariz. Estando en posición, el instructor de la prueba indica que se realice una inspiración máxima, lenta y progresiva que el paciente debe mantener menos de 1 segundo y que posteriormente debe expulsar lo más fuerte y rápidamente posible durante, al menos, 6 segundos. Se verificó que durante la realización de la prueba no existieran fugas alrededor de la boquilla que evitarán los cambios de postura del paciente durante la ejecución.

Por otro lado, el instructor debía vigilar la aparición de eventos durante la prueba, como tos, disnea, dolor torácico y curvas anormales que pueden ser un indicio del uso de broncodilatadores u otros que pueden alterar la respuesta en el desarrollo de la prueba. La prueba se repitió hasta 8 veces con el fin de conseguir 3 ejecuciones técnicamente correctas según los criterios de la American Thoracic Society (ATS).

\section{Análisis estadístico}

Se realizó un análisis descriptivo según la edad, el sexo, el peso, la talla, el hábito tabáquico y los resultados de la espirometría con valores estadísticos de media, desviación típica y varianza. Para la comparación de proporciones, se utilizaron la prueba de $\chi^{2}$ y de McNemar y la prueba exacta de Fisher para medidas repetidas.

Para la comparación de medias, se utilizó la prueba de la $t$ de Student-Fisher para medidas repetidas, o la $t$ de Wilcoxon. El análisis de correlación se determinó mediante la $r$ de Pearson para volúmenes y años de abstinencia de tabaco. Para todas las pruebas, el grado de significación estadística elegido fue de 0,05. El análisis de los datos se realizó mediante el paquete estadístico SPSS versión 2.0.

\section{Resultados}

De los 848 participantes en el estudio, 28 (3,3\%) fueron mujeres (M) y $820(96,7 \%)$ fueron hombres $(\mathrm{H})$, con una edad media de 36,62 $\pm 10,15$ (H: 36,62 $\pm 10,13$ frente a M: 40,36 $\pm 15,86)$ años, una talla de $169,71 \pm 6,77$ (H: 169,71 \pm 6,77 frente a M: 160,32 \pm $6,79)$ y un peso de $72,66 \pm 12,56(\mathrm{H}: 72,66 \pm 12,56$ frente a M: $64,57 \pm 13,20)$. Sobre el hábito y el consumo de tabaco, la media para el tiempo de abstinencia es de 4,34 $\pm 5,27$ años y un antiguo consumo diario relativo de 5,76 \pm 7,28 cigarrillos por día.

Por otra parte, en la Tabla 1 se definen las características del sexo, la talla, el peso, los años de abstinencia de tabaco y la cantidad de consumo relativo; esto se asocia en grupos de edades medias segmentadas en 5 puntos corte, con una amplitud estadística de 17,25.

En la Tabla 2 se describen las capacidades y los volúmenes pulmonares teniendo en cuenta el género; además, se describen los parámetros de los valores espiratorios e inspiratorios teniendo en cuenta que se escogió, dentro de las tres pruebas que cumplían con los criterios, la mejor (best trial).

Sobre los valores espirométricos, la relación de la media de $\mathrm{FEV}_{1} / \mathrm{FVC}$ fue de 87,28\%, $\mathrm{FEV}_{1}$ de 3,81 L 
$\pm 0,02$ y FVC de 4,38 L $\pm 0,02$. En mujeres, los valores fueron de $89,91 \%, 3,06 \mathrm{~L} \pm 0,12$ y $2,76 \mathrm{~L} \pm 0,12$ para $\mathrm{FEV}_{1} / \mathrm{FVC}, \mathrm{FEV}_{1}$ y FVC, respectivamente. En los hombres, los resultados para la relación $\mathrm{FEV}_{1} / \mathrm{FVC}$, la $\mathrm{FEV}_{1}$ y la FVC fueron de $87,19 \%, 4,43 \pm 0,02$ y $3,85 \pm 0,02$.

También se determinaron los valores estadísticos del promedio y el error típico de los valores obtenidos por los participantes con los valores predichos o teóricos. En la Tabla 3 se puede apreciar esquemáticamente que no hay una convergencia entre los resultados reales y los teóricos basados en las características de los sujetos particularmente. La comparación entre medidas, donde se asocia la correlación entre las muestras obtenidas y las predichas, y las diferencias estadísticas se presentan en las Tablas 4 y $\mathbf{5}$.

Tabla 1. Características de la población para la edad agrupada $(n=848)$

\begin{tabular}{|c|c|c|c|c|c|c|c|c|c|}
\hline \multirow{3}{*}{ Características } & \multicolumn{9}{|c|}{ Edad (años) } \\
\hline & \multicolumn{2}{|c|}{$16-33$} & \multicolumn{2}{|c|}{$34-51$} & \multicolumn{2}{|c|}{$52-68$} & \multicolumn{2}{|c|}{$>=69$} & \\
\hline & $n$ & $\overline{\mathbf{x}}$ & $n$ & $\overline{\mathbf{x}}$ & $n$ & $\overline{\mathbf{x}}$ & $n$ & $\overline{\mathbf{x}}$ & \\
\hline \multirow[t]{2}{*}{ Sexo } & Hombres & 356 & & 379 & & 81 & & 4 & \\
\hline & Mujeres & 12 & & 9 & & 5 & & 2 & \\
\hline \multicolumn{2}{|l|}{ Talla (cm) } & 368 & 170 & 388 & 170 & 86 & 166 & 6 & 165 \\
\hline \multicolumn{2}{|l|}{ Peso (kg) } & 368 & 71 & 388 & 74 & 86 & 72 & 6 & 70 \\
\hline \multicolumn{2}{|l|}{ Exfumador (años) } & 368 & 3 & 388 & 5 & 86 & 9 & 6 & 13 \\
\hline \multicolumn{2}{|c|}{ Consumo (unidades*día) } & 368 & 5 & 388 & 6 & 86 & 10 & 6 & 11 \\
\hline
\end{tabular}

$n=$ muestra; $\overline{\mathbf{x}}=$ promedio.

Tabla 2. Capacidades y volúmenes pulmonares según el género $(n=848)$

\begin{tabular}{|c|c|c|c|c|c|c|}
\hline \multirow{3}{*}{$\begin{array}{l}\text { Capacidades y volúmenes } \\
\text { pulmonares }\end{array}$} & \multicolumn{6}{|c|}{ Género } \\
\hline & \multicolumn{3}{|c|}{ Hombres } & \multicolumn{3}{|c|}{ Mujeres } \\
\hline & $\overline{\mathbf{x}}$ & & $\sigma$ & $\overline{\mathbf{x}}$ & & $\sigma$ \\
\hline FVC & 4,43 & \pm & ,02 & 3,06 & \pm & ,12 \\
\hline $\mathrm{FEV}_{1}$ & 3,85 & \pm & ,02 & 2,76 & \pm & ,12 \\
\hline PEF & 8,04 & \pm & ,05 & 5,46 & \pm & ,27 \\
\hline $\mathrm{FEF}_{75-}$ & 2,31 & \pm & ,03 & 1,88 & \pm & ,13 \\
\hline $\mathrm{FEF}_{25-75}$ & 4,49 & \pm & ,04 & 3,40 & \pm & , 18 \\
\hline FET & 2,41 & \pm & ,03 & 2,05 & \pm & ,11 \\
\hline $\mathrm{FEV}_{1 \_} \mathrm{FVC}$ & 87,19 & \pm & ,21 & 89,91 & \pm & 1,10 \\
\hline $\mathrm{FEV}_{6}$ & 4,43 & \pm & ,02 & 3,06 & \pm & ,12 \\
\hline $\mathrm{FEV}_{3}$ & 4,40 & \pm & ,02 & 3,06 & \pm & ,12 \\
\hline $\mathrm{FEV}_{1-\mathrm{FEV}_{6}}$ & 87,19 & \pm & ,21 & 89,91 & \pm & 1,10 \\
\hline $\mathrm{FEF}_{25}$ & 7,23 & \pm & ,05 & 5,06 & \pm & ,24 \\
\hline
\end{tabular}


Tabla 2. Capacidades y volúmenes pulmonares según el género $(n=848)$ (Continuación)

\begin{tabular}{|c|c|c|c|c|c|c|}
\hline \multirow{3}{*}{$\begin{array}{l}\text { Capacidades y volúmenes } \\
\text { pulmonares }\end{array}$} & \multicolumn{6}{|c|}{ Género } \\
\hline & \multicolumn{3}{|c|}{ Hombres } & \multicolumn{3}{|c|}{ Mujeres } \\
\hline & $\overline{\mathbf{x}}$ & & $\sigma$ & $\overline{\mathbf{x}}$ & & $\sigma$ \\
\hline $\mathrm{FEF}_{50}$ & 4,97 & \pm &, 04 & 3,64 & \pm & ,20 \\
\hline FIV1 & 3,54 & \pm & ,05 & 2,29 & \pm & 18, \\
\hline FIV $_{1}$ FIVC & 3,36 & \pm &, 05 & 2,22 & \pm & , 18 \\
\hline PIF & 86,10 & \pm & 1,02 & 86,61 & \pm & 5,88 \\
\hline $\mathrm{FEV}_{1 \_\mathrm{VC}}$ & 4,24 & \pm &, 07 & 3,00 & \pm & ,28 \\
\hline $\mathrm{FEV}_{3} \mathrm{FVC}$ & ,00 & \pm & ,00 & ,00 & \pm & ,00 \\
\hline
\end{tabular}

$\overline{\mathrm{x}}=$ promedio; $\sigma$ = error típico; $\mathrm{FEV}_{3}=$ volumen espiratorio máximo forzado a los 3 segundos; $\mathrm{FEV}_{6}=$ volumen espiratorio máximo forzado a los 6 segundos; $\mathrm{FEF}_{25}=$ flujo espiratorio forzado del $25 \%$; $\mathrm{FEF}_{50}=$ flujo espiratorio forzado del $50 \%$; $\mathrm{FEF}_{75}=$ flujo espiratorio forzado del $75 \% ; \mathrm{FEF}_{25-75}=$ flujo espiratorio forzado entre el 25-75\%; $\mathrm{FET}$ = tiempo de espiración forzada; FIVC = capacidad vital forzada inspiratoria; FIV $_{1}=$ Volumen Inspiratorio Máximo Forzado al primer segundo; $\mathrm{PIF}=$ flujo inspiratorio máximo.

Tabla 3. Capacidades y volúmenes pulmonares frente a valores predichos $(n=848)$

\begin{tabular}{|c|c|c|c|c|c|c|}
\hline \multirow{2}{*}{$\begin{array}{l}\text { Capacidades y volúmenes } \\
\text { pulmonares }\end{array}$} & \multicolumn{3}{|c|}{ Valores reales } & \multicolumn{3}{|c|}{ Valores predichos } \\
\hline & $\overline{\mathbf{x}}$ & & $\sigma$ & $\overline{\mathbf{x}}$ & & $\sigma$ \\
\hline FVC & 4,38 & \pm & ,02 & 4,49 & \pm & ,02 \\
\hline $\mathrm{FEV}_{1}$ & 3,81 & \pm & ,02 & 3,76 & \pm & ,02 \\
\hline PEF & 7,96 & \pm & ,05 & 8,98 & \pm &, 03 \\
\hline $\mathrm{FEF}_{75-}$ & 2,29 & \pm & ,03 & 2,16 & \pm & ,01 \\
\hline $\mathrm{FEF}_{25-75}$ & 4,45 & \pm & ,04 & 4,44 & \pm & ,02 \\
\hline FET & 2,40 & \pm & ,03 & 6,00 & \pm & ,00 \\
\hline $\mathrm{FEV}_{1 \_} \mathrm{FVC}$ & 87,28 & \pm & ,21 & 80,92 & \pm & ,06 \\
\hline $\mathrm{FEV}_{6}$ & 4,38 & \pm & ,02 & 4,49 & \pm & ,02 \\
\hline $\mathrm{FEV}_{3}$ & 4,36 & \pm & ,02 & 4,27 & \pm & ,02 \\
\hline $\mathrm{FEV}_{1} \mathrm{FEV}_{6}$ & 87,28 & \pm & ,21 & 83,74 & \pm & ,06 \\
\hline $\mathrm{FEF}_{25}$ & 7,16 & \pm & ,05 & 7,73 & \pm & ,02 \\
\hline $\mathrm{FEF}_{50}$ & 4,92 & \pm & ,04 & 4,96 & \pm & ,02 \\
\hline $\mathrm{FIV}_{1}$ & 3,50 & \pm &, 04 & 4,49 & \pm & ,02 \\
\hline FIV $_{1}$ FIVC & 3,32 & \pm & ,05 & 3,76 & \pm & ,02 \\
\hline PIF & 86,12 & \pm & 1,00 & 80,92 & \pm & ,06 \\
\hline $\mathrm{FEV}_{1-\mathrm{VC}}$ & 4,20 & \pm & ,07 & 8,98 & \pm & ,03 \\
\hline $\mathrm{FEV}_{3-} \mathrm{FVC}$ &, 00 & \pm & ,00 & 80,92 & \pm & ,06 \\
\hline
\end{tabular}


Tabla 4. Correlaciones de muestras relacionadas $(n=848)$

\begin{tabular}{|c|c|c|c|}
\hline Capacidades y volúmenes pulmonares & $n$ & Correlación & Sig. \\
\hline FVC y FVC_P & 848 & ,653 &, 000 \\
\hline $\mathrm{FEV}_{1}$ y $\mathrm{FEV}_{1-} \mathrm{P}$ & 848 & ,665 & ,000 \\
\hline PEF y PEF_P & 848 & ,391 &, 000 \\
\hline $\mathrm{FEF}_{75-}$ y $\mathrm{FEF}_{75-} \mathrm{P}$ & 848 & ,342 & ,000 \\
\hline $\mathrm{FEF}_{25-75}$ y $\mathrm{FEF}_{25-75-} \mathrm{P}$ & 848 & ,396 &, 000 \\
\hline FET y FET_P & 848 & & \\
\hline $\mathrm{FEV}_{1}$ FVC y FEV 1 FVC_P & 848 & , 171 &, 000 \\
\hline $\mathrm{FEV}_{6}$ y $\mathrm{FEV}_{6-} \mathrm{P}$ & 848 & ,653 &, 000 \\
\hline $\mathrm{FEV}_{3}$ y $\mathrm{FEV}_{3-} \mathrm{P}$ & 848 & ,635 &, 000 \\
\hline $\mathrm{FEV}_{1-} \mathrm{FEV}_{6}$ y $\mathrm{FEV}_{1} \mathrm{FEV}_{6-} \mathrm{P}$ & 848 & ,235 &, 000 \\
\hline $\mathrm{FEF}_{25}$ y $\mathrm{FEF}_{25-} \mathrm{P}$ & 848 & ,382 & ,000 \\
\hline $\mathrm{FEF}_{50}$ y $\mathrm{FEF}_{50-} \mathrm{P}$ & 848 & ,348 &, 000 \\
\hline $\mathrm{FIV}_{1}$ y FIV $1-\mathrm{P}$ & 848 & ,287 &, 000 \\
\hline 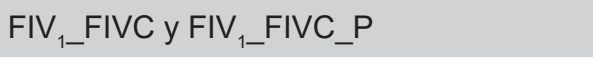 & 848 & ,249 &, 000 \\
\hline PIF y PIF_P & 848 &,- 051 & , 138 \\
\hline $\mathrm{FEV}_{1-\mathrm{VC} \text { y FEV } 1-V C \_P}$ & 848 & ,181 &, 000 \\
\hline $\mathrm{FEV}_{3-} \mathrm{FVC}$ y $\mathrm{FEV}_{3} \mathrm{FVC}_{-} \mathrm{P}$ & 848 & & \\
\hline
\end{tabular}

FVC_P = capacidad vital forzada predicha; $F E V_{1} \mathrm{P}=$ volumen espiratorio máximo forzado al primer segundo predicho; $\mathrm{FEV}_{6-} \mathrm{P}=$ volumen espiratorio máximo forzado a los 3 segundos predicho; $F E V_{6-} \mathrm{P}=$ volumen espiratorio máximo forzado a los 6 segundos predicho; $P E F=$ flujo espiratorio máximo; $P E F \_P=$ flujo espiratorio máximo predicho; $F E F_{25} P=$ flujo espiratorio forzado del $25 \%$ predicho; $\mathrm{FEF}_{50} \mathrm{P}=$ flujo espiratorio forzado del $50 \%$ predicho; $\mathrm{FEF}_{75-} \mathrm{P}=$ flujo espiratorio forzado

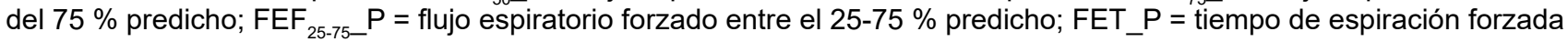
predicha; FIVC_P = capacidad vital forzada inspiratoria predicho; FIV 1 P = volumen inspiratorio máximo forzado al primer segundo predicho; PIF_P = flujo inspiratorio máximo predicho.

Tabla 5. Prueba de muestras relacionadas $(n=848)$

\begin{tabular}{|c|c|c|c|c|c|c|c|}
\hline \multirow{3}{*}{$\begin{array}{l}\text { Capacidades y } \\
\text { volúmenes pulmonares }\end{array}$} & \multicolumn{7}{|c|}{ Diferencias relacionadas } \\
\hline & \multirow[t]{2}{*}{$\overline{\mathbf{x}}$} & \multirow[t]{2}{*}{$\sigma$} & \multicolumn{2}{|c|}{$\begin{array}{c}\text { Intervalo de confianza (IC) } \\
\text { del } 95 \%\end{array}$} & \multirow{2}{*}{$t$} & \multirow{2}{*}{ gl } & \multirow{2}{*}{$\begin{array}{c}\text { Sig. } \\
\text { (bilateral }\end{array}$} \\
\hline & & & Inferior & Superior & & & \\
\hline FVC-FVC_P &,- 10960 & ,01807 &,- 14506 &,- 07413 & $-6,066$ & 847 &, 000 \\
\hline $\mathrm{FEV}_{1}-\mathrm{FEV}_{1} \mathrm{P}$ & ,04974 & ,01500 & ,02029 & ,07919 & 3,316 & 847 & ,001 \\
\hline PEF-PEF_P & $-1,02526$ & ,05065 & $-1,12468$ &,- 92584 & $-20,242$ & 847 & ,000 \\
\hline $\mathrm{FEF}_{75-}-\mathrm{FEF}_{75-} \mathrm{P}$ & ,12783 & ,02543 & ,07792 & , 17774 & 5,027 & 847 &, 000 \\
\hline $\mathrm{FEF}_{25-75}-\mathrm{FEF}_{25-75-} \mathrm{P}$ &, 00817 & ,03324 &,- 05708 & ,07342 & ,246 & 847 & ,806 \\
\hline FET-FET_P & $-3,60289$ & ,02996 & $-3,66169$ & $-3,54409$ & $-120,263$ & 847 &, 000 \\
\hline
\end{tabular}


Tabla 5. Prueba de muestras relacionadas $(n=848)$ (Continuación)

\begin{tabular}{|c|c|c|c|c|c|c|c|}
\hline \multirow{3}{*}{$\begin{array}{l}\text { Capacidades y } \\
\text { volúmenes pulmonares }\end{array}$} & \multicolumn{7}{|c|}{ Diferencias relacionadas } \\
\hline & \multirow{2}{*}{$\overline{\mathbf{x}}$} & \multirow{2}{*}{$\sigma$} & \multicolumn{2}{|c|}{$\begin{array}{c}\text { Intervalo de confianza (IC) } \\
\text { del } 95 \%\end{array}$} & \multirow{2}{*}{$\mathbf{t}$} & \multirow{2}{*}{ gl } & \multirow{2}{*}{$\begin{array}{c}\text { Sig. } \\
\text { (bilateral) }\end{array}$} \\
\hline & & & Inferior & Superior & & & \\
\hline $\mathrm{FEV}_{1}$ FVC-FEV 1 FVC_P & 6,35948 & ,20861 & 5,95002 & 6,76894 & 30,485 & 847 & ,000 \\
\hline $\mathrm{FEV}_{6}-\mathrm{FEV}_{6-} \mathrm{P}$ &,- 10969 & 01807 &,- 14515 &,- 07423 & $-6,072$ & 847 &, 000 \\
\hline $\mathrm{FEV}_{3}-\mathrm{FEV}_{3-} \mathrm{P}$ & 09025 & ,01851 & ,05391 & ,12659 & 4,875 & 847 &, 000 \\
\hline $\mathrm{FEV}_{1} \mathrm{FEV}_{6}-\mathrm{FEV}_{1-} \mathrm{FEV}_{6-} \mathrm{P}$ & 3,5384 & ,20464 & 3,13674 & 3,94006 & 17,291 & 847 &, 000 \\
\hline $\mathrm{FEF}_{25}-\mathrm{FEF}_{25-} \mathrm{P}$ &,- 56940 &, 04629 &,- 66026 &,- 47854 & $-12,301$ & 847 &, 000 \\
\hline $\mathrm{FEF}_{50}-\mathrm{FEF}_{50-} \mathrm{P}$ &,- 04316 & ,03788 &,- 11750 & ,03118 & $-1,140$ & 847 &, 255 \\
\hline $\mathrm{FIV}_{1}-\mathrm{FIV}_{1-} \mathrm{P}$ &,- 99072 & ,04337 & $-1,07584$ &,- 90560 & $-22,844$ & 847 & ,000 \\
\hline FIV $_{1}$ FIVC-FIV ${ }^{1}$ FIVC_P &,- 43976 & ,04443 &,- 52697 &,- 35256 & $-9,898$ & 847 &, 000 \\
\hline PIF-PIF_P & 5,19651 & 1,0082 & 3,21761 & 7,17541 & 5,154 & 847 &, 000 \\
\hline FEV 1 VC-FEV 1 VC_P & $-4,78047$ & ,06790 & $-4,91374$ & $-4,64720$ & $-70,407$ & 847 &, 000 \\
\hline $\mathrm{FEV}_{3} \mathrm{FVC}_{-} \mathrm{FEV}_{3-} \mathrm{FVC} \mathrm{P}$ & $-80,9196$ & ,06053 & $-81,0384$ & $-80,8008$ & $-1336,92$ & 847 & ,000 \\
\hline
\end{tabular}

De los sujetos, 754 (88,92 \%) tenían valores de $\mathrm{FEV}_{1} / \mathrm{FVC}$ "normales” y $54(6,37 \%)$ presentaban obstrucción pulmonar leve tras haber dejado el hábito de consumo de tabaco por más de un año.

\section{Discusión}

En este estudio se describen las capacidades y los volúmenes pulmonares de sujetos exfumadores de más de 15 empresas del sector industrial de la ciudad de Cúcuta, Colombia. La investigación incluyó profesionales, técnicos, académicos y empleados de oficios varios.

La agrupación por edades permitió describir poblaciones desde los 16 hasta los 85 años. Los criterios de selección definidos a priori, la técnica de muestreo en forma probabilística y por reemplazo, así como la verificación del cumplimiento de los criterios de inclusión, nos permiten decir con adecuada confiabilidad que el estudio se adelantó en una muestra de sujetos exfumadores y en abstinencia del consumo tabáquico.

A partir de lo anterior, este estudio coincide con los estudios realizados por la ATS sobre la divergencia de valores para la espirometría en los sujetos con características antropométricas relativamente cercanas (26).

Clotet y colaboradores (27) realizaron un estudio con espirometría como método de tamizaje e intervención de fumadores de alto riesgo en 497 sujetos en 40 y 70 años. Se encontró que son los hombres quienes más consumen tabaco en edades más adultas y en cantidades superiores a las dos unidades por día, así como lo demuestra esta investigación.

De otra manera, Reyes y colaboradores (28) realizaron un estudio descriptivo de corte transversal en trabajadores fumadores; estudiaron 60 individuos fumadores, a quienes se les realizó un estudio espirométrico para evaluar la función pulmonar. Los autores encontraron un mayor predominio de población femenina entre los 40 y 49 años con hábito tabáquico, con un consumo diario de 11-20 unidades de cigarrillos.

Por otro lado, un estudio observacional realizado por Muñoz-Pérez y colaboradores (29), con 160 sujetos entre los 18 a 25 años con población fumadora y no fumadora, pretendía identificar la obstrucción de las 
vías aéreas a partir de la espirometría. Los fumadores obtuvieron una media en la relación $\mathrm{FEV}_{1} / \mathrm{FVC}$ de $87,3 \pm 9,27$ que reveló una diferencia estadísticamente significativa ( $p=0,0045)$ con el grupo control (no fumadores); la espirometría arrojó datos de obstrucción leve en 19 participantes con una media de relación $\mathrm{FEV}_{1} / \mathrm{FVC}$ de $74,5 \pm 4,34$.

Otro estudio dirigido por Martínez-López y colaboradores (30) buscó evaluar la función pulmonar de 800 sujetos segmentados sociodemográficamente mediante espirometría, teniendo en cuenta la exposición al hábito tabáquico. En todos los sujetos expuestos al tabaco se encontraron niveles de capacidades y volúmenes pulmonares reducidos de un $32,5 \%$, con un odds ratio [OR] de 2,09 (IC del 95 \%: 1,49 a 2,42; $\chi^{2}$ al cuadrado: $20,2 ; p=0,00)$. Asimismo, diferentes investigaciones (31-33) muestran la asociación entre la edad y el consumo de tabaco con la predicción en la estimación de los valores espirométricos, con descensos marcados por la obstrucción del flujo aéreo.

\section{Conclusiones}

El consumo del tabaco reduce los parámetros de las capacidades y los volúmenes pulmonares en las personas, independientemente de las características antropométricas. A pesar de ser exfumadores, se encontró una baja prevalencia de deterioro pulmonar tras haber cesado el hábito de tabaquismo, lo cual podría suponer que el cese del consumo de cigarrillo podría revertir las afectaciones generadas por este hábito.

\section{Financiación}

No hubo financiamiento por ninguna entidad.

\section{Conflicto de intereses}

Los autores declaran no tener ningún conflicto de intereses.

\section{Referencias}

1. Dempsey TM, Scanlon PD. Pulmonary Function Tests for the Generalist: A Brief Review. Mayo Clin Proc. 2018;93(6):763771. doi:10.1016/j.mayocp.2018.04.009
2. Dancer R, Thickett, D. Assessment of pulmonary function. Medicine, 2016;44(4):226-9. doi:10.1016/j.mpmed.2016.02.007

3. World Health Organization [Internet]. Geneva: WHO; 2007. Global surveillance, prevention and control of chronic respiratory diseases. A comprehensive approach. Disponible en: http://www.who.int/gard/publications/GARD_Manual/en/

4. Burney PG, Patel J, Newson R, Minelli C, Naghavi M. Global and regional trends in COPD mortality, 1990-2010. Eur Respir. 2015;45(5):1239-1247. doi:10.1183/09031936.00142414

5. World Health Organization [Internet]. Luxemburgo: World Health Organization; 2015. WHO report on the global tobacco epidemic, 2015: raising taxes on tobacco. Disponible en: https://apps.who.int/iris/bitstream/handle/10665/178574/9789240694606_eng.pdf;jsessionid=1F005 E6C35C8001D3B8EE060C75B0545? sequence $=1$

6. Talaminos-Barroso A, Márquez-Martín E, Roa-Romero LM, Ortega-Ruiz F. Factores que afectan a la función pulmonar: una revisión bibliográfica. Arch Bronconeumol. 2018;54(6): 327-32. doi:10.1016/j.arbres.2018.01.030

7. Scanlon PD. Respiratory function: mechanisms and testing. En: Goldman L, Schafer AI, editores. Goldman-Cecil Medicine. 25. ${ }^{\text {a }}$ ed. Philadelphia, PA: Elsevier Saunders. 2016. p. $539-45$.

8. Álvarez FJ, Barchilón V, Casas F, Compán MV, Entrenas LM, Fernández J, et al. Documento de Consenso sobre la espirometría en Andalucía. SEMERGEN. 2009;35(9):457-68. doi: 10.1016/S1138-3593(09)72845-X

9. Hwang JW, Sundar IK, Yao H, Sellix MT, Rahman I. Circadian clock function is disrupted by environmental tobacco/ cigarette smoke, leading to lung inflammation and injury via a SIRT1-BMAL1 pathway. FASEB J. 2014;28(1):176-94. doi:10.1096/fj.13-232629

10. Mottram C. Ruppel's Manual of Pulmonary Function Testing. 10. ${ }^{a}$ edición. Mary-land Heights, Missouri: Mosby; 2012. p. 3.

11. Farha S, Asosingh K, Laskowski D, Licina L, Sekiguchi H, Sekigushi $\mathrm{H}$, et al. Pulmonary gas transfer related to markers of angiogenesis during the menstrual cycle. J Appl Physiol, 2007;103(5):1789-95. doi:10.1152/japplphysiol.00614.2007

12. Löfstedt $H$, Hagström $K$, Bryngelsson IL, Holmström M, Rask-Andersen A. Respiratory symptoms and lung function in relation to wood dust and monoterpene exposure in the wood pellet industry. Ups J Med Sci. 2017;122(2):78-84. doi :10.1080/03009734.2017.1285836

13. Bowatte G, Lodge CJ, Knibbs LD, Lowe AJ, Erbas B, Dennekamp M, et al. Traffic related air pollution exposure is associated with allergic sensitization, asthma, and poor lung function in middle age. J Allergy Clin Immunol. 2017;139(1):122-9. e1. doi:10.1016/j.jaci.2016.05.008

14. Rice MB, Li W, Wilker EH, Gold DR, Schwartz J, Koutrakis $\mathrm{P}$, et al. Extreme temperatures and lung function in the Framingham Heart Study. A59 Epidemiology of Airways and Chronic Lung Disesases. 2017;A2042-A2042.12. 
15. Kobayashi S, Hanagama M, Yamanda S, Satoh H, Tokuda S, Kobayashi M, et al. Impact of a large-scale natural disaster on patients with chronic obstructive pulmonary disease: The aftermath of the 2011 Great East Japan Earthquake. Respir Investig. 2013;51(1):17-23. doi:10.1016/j.resinv.2012.10.004

16. Donnelly PM, Yang TS, Peat JK, Woolcock AJ. What factors explain racial differences in lung volumes? Eur Respir J. 1991;4(7):829-38.

17. Barone-Adesi F, Dent JE, Dajnak D, Beevers S, Anderson HR, Kelly FJ, et al. Long-term exposure to primary traffic pollutants and lung function in children: Cross-sectional study and meta-analysis. PLoS One. 2015;10(11):e0142565. doi:10.1371/journal.pone.0142565

18. Tabak C, Spijkerman AM, Verschuren WM, Smit HA. Does educational level influence lung function decline (Doetinchem Cohort Study)? Eur Respir J. 2009;34(4):940-7. doi:10.1183/09031936.00111608

19. Lange P, Marott JL, Vestbo J, Ingebrigtsen TS, Nordestgaard BG. Socioeconomic status and prognosis of COPD in Denmark. COPD. 2014;11(4):431-7. doi:10.3109/15412555.201 3.869580

20. Dane DM, Lu H, Dolan J, Thaler CD, Ravikumar P, Hammond KA, et al. Lung function and maximal oxygen uptake in deer mice (Peromyscus maniculatus) bred at low altitude and re-acclimatized to high altitude. FASEB J. 2016;30,1297.51297. doi: 10.1096/fasebj.30.1_supplement.1297.5

21. Mehari A, Afreen S, Ngwa J, Setse R, Thomas AN, Poddar $\mathrm{V}$, et al. Obesity and Pulmonary Function in African Americans. PLoS One. 2015;10:e0140610. doi:10.1371/journal. pone. 0140610

22. Shan X, Liu J, Luo Y, Xu X, Han Z, Li H. Relationship between nutritional risk and exercise capacity in severe chronic obstructive pulmonary disease in male patients. Int J Chron Obstruct Pulmon Dis. 2015;10:1207-12. doi:10.2147/COPD. S82082

23. Lazovic B, Mazic S, Suzic-Lazic J, Djelic M, Djordjevic-Saranovic S, Durmic T, et al. Respiratory adaptations in different types of sport. Eur Rev Med Pharmacol Sci. 2015;19(12):2269-74.

24. Hoesein FA, Jong PA, Lammers JW, Mali WP, Mets OM, Schmidt $\mathrm{M}$, et al. Contribution of CT quantified emphyse- ma, air trapping and airway wall thick-ness on pulmonary function in male smokers with and without COPD. COPD. 2014;11(5):503-9. doi:10.3109/15412555.2014.933952

25. Gouna G, Rakza T, Kuissi E, Pennaforte T, Mur S, Storme L. Positioning effects on lung function and breathing pattern in premature newborns. J Pediatr. 2013;162(6):1133-7. doi:10.1016/j.jpeds.2012.11.036

26. Standardization of Spirometry, 1994 Update. American Thoracic Society. Am J Respir Crit Care Med. 1995;152(3):11071136. doi:10.1164/ajrccm.152.3.7663792

27. Clotet J, Gómez X, Ciria C, Albalad JM. La espirometría es un buen método para la detección y el seguimiento de la EPOC en fumadores de alto riesgo en atención primaria. Arch Bronconeumol. 2004;40(4):155-9. doi: 10.1016/S03002896(04)75495-2

28. Reyes D, García M, Simón P, Pérez K. Repercusión del hábito de fumar en la función pulmonar de fumadores activos. Rev Cub Med Mil. 2011;40(3-4), 227-33.

29. Muñoz-Pérez MJ, Palafox D, Palafox J, Vichido-Luna MA, Espinosa N, Rivas-Chávez A. Determinación de valores espirométricos en jóvenes fumadores y no fumadores. Med Int Mex. 2013;29(6):553-557.

30. Martínez-López E, Díaz PA. Tabaquismo y disminución de la función pulmonar en hombres y mujeres adultos. Salud(i) ciencia. 2013;20(3):246-250.

31. Puhan MA, García-Aymerich J, Frey M, ter Riet G, Anto JM, Agusti AG, et al. Expansion of the prognostic assessment of patients with chronic obstructive pulmonary disease: the updated BODE index and the ADO index. Lancet. 2009;374(9691):704-11. doi:10.1016/S0140-6736(09)613015

32. Soler-Cataluña JJ, Martínez-García MA, Sánchez LS, Tordera MP, Sánchez PR. Severe exacerbations and BODE index: two independent risk factors for death in male COPD patients. Respir Med. 2009;103(5):692-9. doi:10.1016/j.rmed.2008.12.005

33. Schembri S, Anderson W, Morant S, Winter J, Thompson P, Pettitt D, et al. A predictive model of hospitalisation and death from chronic obstructive pulmonary disease. Respir Med. 2009;103(10):1461-7. doi:10.1016/j.rmed.2009.04.021 